\title{
TUNABLE SEALED-OFF CW CO LASER AT ROOM TEMPERATURE
}

\author{
P.J.M. PETERS, W.J. WITTEMAN and Z. KRZYZANOWSKI \\ Department of Applied Physics, Twente University of Technology, Enschede, The Netherlands
}

Received 2 November 1982

Revised manuscript received 29 December 1982

\begin{abstract}
Starting from a sealed-off CO laser with a long-life output of $29 \mathrm{~W} / \mathrm{m}$ in multiline operation, we developed a tunable version of this device. The system described has a discharge length of $97 \mathrm{~cm}$. It was made transition-selective by using a three-mirror configuration. In this way we were able to tune the laser to more than 70 oscillating vibrational-rotational transitions of the $\mathrm{CO}$ molecule.
\end{abstract}

There is an increasing need for broadband tunable lasers, with a narrow line-width, in the near-infrared region. Especially spectroscopists are interested in the region around $5 \mu \mathrm{m}$, but also for laser-induced chemistry and air-pollution monitoring there are interesting applications for lasers in this wavelength region.

A tunable cw $\mathrm{CO}$ laser will be a very attractive candidate because of its high output power and the large number of potential laser lines. It is possible to cover with this laser the spectral range from about $5 \mu \mathrm{m}$ up to $8 \mu \mathrm{m}$. To have a stable and high output power, many investigators use $\mathrm{cw} \mathrm{CO}$ lasers that are cooled down to liquid-nitrogen temperature and with continuously flowing gas mixtures.

It will be clear that a sealed-off CO laser, working at room temperature, would be much easier to work with and to transport from one place to another.

Many attempts have been made to develop such a sealed-off laser. Now it is generally believed that for long-life operation the elimination of hydrogen or waver vapour is essential.

In 1980 we reported on a solution for this problem by using gold electrodes and a quartz discharge tube that was provided with a small cell containing zeolite [1]. The zeolite is used as an absorber of any water that is either released from the construction materials or produced in the discharge from released hydrogen. With this sealed-off tube working at room temperature we got a multiline output power of $29 \mathrm{~W} / \mathrm{m}$. After one year of regular use the output power of this tube was still $22.5 \mathrm{~W} / \mathrm{m}$.

In this paper we will report on our recent experiments for developing a tunable laser starting from the sealed-off $\mathrm{CO}$ laser mentioned above. Several techniques can be used to make a cw laser wavelength-selec tive. We chose a three-mirror configuration, which can yield single-mode emission. The principles of this three-mirror device have been described for $\mathrm{cw} \mathrm{CO}_{2}$ lasers by Ernst and Witteman in 1971 [2]. It consists of an output coupling window with a suitable chosen radius of curvature on one side and on the other side of the laser tube a Fabry-Perot etalon. The Fabry. Perot etalon has one broad-band reflector and a rotatable wavelength-selective grating.

Using this three-mirror configuration, we developed in the past a cw sealed-off $\mathrm{CO}_{2}$ laser, with a discharge length of about $90 \mathrm{~cm}$. This system met some special requirements. The first one was that the laser should give a very stable output power for each of the selected laser lines. The second requirement was to develop a system that was easily transportable from one place to another without any need for re-adjustments of either the mirror or the grating. The system designed appeared to be tunable over more than 80 transitions in both the $P$ and $R$ branches of the $00^{0} 1-$ $10^{\circ} 0$ and $00^{0} 1-02^{0} 0$ vibrational bands of the $\mathrm{CO}_{2}$ molecule. From experiments with this $\mathrm{CO}_{2}$ laser we could conclude that the design came up to our expectations. 


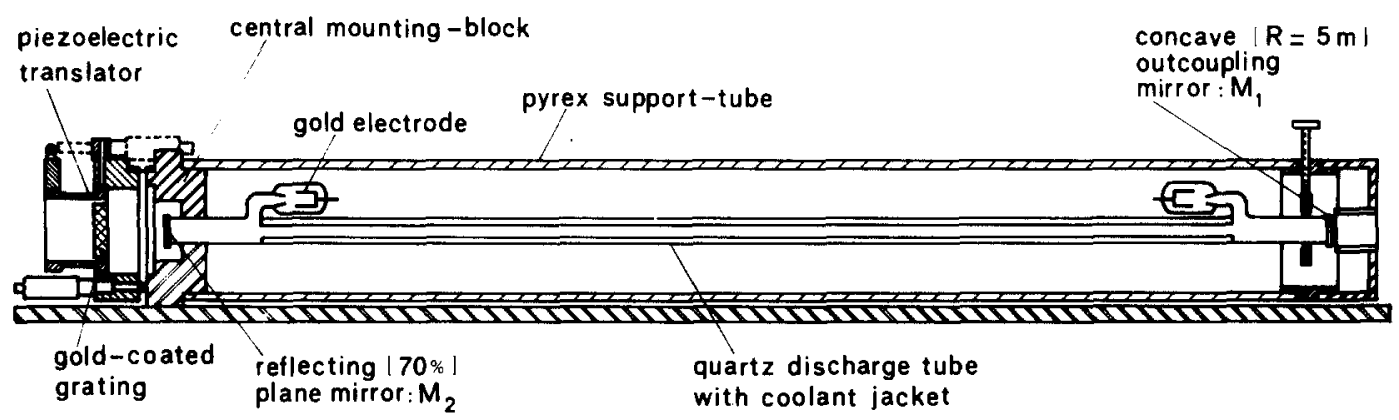

Fig. 1. Cross-section of the tunable CO laser.

The same system we now investigated with a sealed-off $\mathrm{CO}$ laser. The experimental set-up is shown in fig. 1 .

Inside a pyrex support tube the quartz discharge tube has been mounted. On-one side the tube has been glued in the central mounting block. In this solid block also the grating has been mounted, with a possibility to turn the grating very precisely around a vertical axis perpendicular to the optical axis. In our measurements we used a 3600 l/inch gold-coated grating, blazed at $5.4 \mu \mathrm{m}$, from Photo Technical Research Inc. The grating was mounted on a piezo-electric translator, in order to tune the length of the Fabry-Perot on the oscillating wave-length. The quartz discharge tube is on the other side, on which the outcoupling mirror has been glued, connected to the pyrex support tube, by means of a slightly adjustable support structure. It should be noticed that the pyrex support tube has been fixed only on one side to the central mounting block. This block, on its turn, has been mounted to a thick, rigid, iron profile. The laser tube had a discharge length of about 90 $\mathrm{cm}$ and an internal diameter of $6 \mathrm{~mm}$. As electrode material we used gold.

For the spectral analysis of the laser output radiation, the beam was chopped and then passed through a microprocessor driven Hilger \& Watts monochromator, which was equipped with a $150 \mathrm{l} / \mathrm{mm}$ grating blazed at $5.4 \mu \mathrm{m}$. The output radiation from the monochromator was detected by an InSb detector and this signal was fed into a lock-in amplifier. The output of the lock-in amplifier can be recorded on an Y-T recorder. The resolving power of our arrangement was about $8 \AA$.

We investigated two different optical configura- tions. In both cases we used on the outcoupling side a germanium window with a reflectivity of $88 \%$. The transmission of this window was about $10 \%$ and the radius of curvature was $5 \mathrm{~m}$. On the other side of the tube we used two different germanium windows, one with a reflectivity of $90 \%$ and the other with a reflectivity of $70 \%$.

When the grating was blocked, both configurations nevertheless emitted laser radiation. According to gain measurements carried out by Bhaumik and colleagues [3] this should be impossible. They estimated that the gain did not exceed $0.2 \% \mathrm{~cm}^{-1}$ in a similar $\mathrm{CO}$ laser. In our tube with the highest losses the maximum gain clearly exceeded their measured values. We concluded this from the fact that the laser threshold value was $0.27 \% \mathrm{~cm}^{-1}$ in our laser and still there were at least five oscillating transitions. By comparing all oscillating rotational-vibrational transitions in multiline operation, we see a remarkable difference for the two optical configurations described. Looking at the lines with the highest intensity, we observe a shift to lower vibrational bands and higher rotational numbers when more transitions oscillate at the same time. We have seen this effect also in a pulsed transversely excited $\mathrm{CO}$ laser working at cryogenic temperatures [4]. It is due to an intrinsic feature of the CO laser: the cascade effect.

With the grating not blocked we found much more oscillating transitions in both configurations. With the window of $90 \%$ reflectivity (at the FabryPerot side) we found only 10 to 15 new lines. This is due to the fact that most of the vibrational energy, which is available in potentially lasing levels, is actually emitted as multiline stimulated radiation because of the low laser-threshold values. So the tuning effect 
Table 1

Measured vibrational-rotational transitions in a wavelength-selective, sealed $-\mathrm{ff}$, cw CO laser $(T=300 \mathrm{~K})$. Note: lines with the same subscript coincide within $0.5 \mathrm{~cm}^{-1}$

\begin{tabular}{|c|c|c|c|c|c|c|c|c|c|c|}
\hline \multirow{2}{*}{$\frac{\text { Vibrational band }}{8 \rightarrow 7}$} & \multicolumn{10}{|c|}{ P-branch transition } \\
\hline & 17 & 18 & 19 & 20 & 21 & 22 & $23 \mathrm{~A}$ & & & \\
\hline $9 \rightarrow 8$ & $17 \mathrm{~A}$ & 18 & 19 & 20 & 21 & $22{ }_{B}$ & $23_{\mathrm{C}}^{\mathrm{A}}$ & & & \\
\hline $10 \rightarrow 9$ & $16_{\mathrm{B}}^{\mathrm{A}}$ & ${ }^{17} \mathrm{C}$ & 18 & 19 & 20 & $21^{\mathrm{B}}$ & $22 \mathrm{D}$ & ${ }^{23} \mathrm{E}$ & & \\
\hline $11 \rightarrow 10$ & $14^{\mathrm{D}}$ & 15 & $16 \mathrm{D}$ & $17 \mathrm{E}$ & 18 & 19 & $20^{D}$ & $21^{\mathrm{E}}$ & $22 \mathrm{~F}$ & ${ }^{23} \mathrm{G}$ \\
\hline $12 \rightarrow 11$ & 14 & & $16_{\mathrm{F}}$ & $17_{\mathrm{G}}^{\mathrm{C}}$ & 18 & 19 & 20 & 21 & & \\
\hline $13 \rightarrow 12$ & 13 & 14 & $15^{1}$ & & 17 & 18 & 19 & 20 & & \\
\hline $14 \rightarrow 13$ & 14 & 15 & 16 & & 17 & 18 & 19 & 20 & 21 & 22 \\
\hline $15 \rightarrow 14$ & 14 & 15 & 16 & & 18 & 19 & 20 & & & \\
\hline $16 \rightarrow 15$ & 14 & & 16 & 17 & 18 & & & & & \\
\hline $17 \rightarrow 16$ & 13 & 14 & & 16 & 17 & 18 & & & & \\
\hline $18 \rightarrow 17$ & 15 & 16 & & & & & & & & \\
\hline
\end{tabular}

of the grating is almost restricted to these transitions, which are either adjacent to the oscillating lines or the next step in the cascade chain. This has been confirmed in our experiments.

If the threshold is much higher, like in our second configuration, the situation is completely different, since there are only a few remaining oscillating transitions.

It should be noted that, in both configurations, if the system is tuned at a particular transition with the grating, we always observe at the same time some additional oscillating transitions. These transitions are generated in the cavity formed by the mirrors $M_{1}$ and $\mathrm{M}_{2}$ and it will be clear that in our second configuration the gain of these transitions will exceed $0.27 \%$ $\mathrm{cm}^{-1}$. With $\mathrm{M}_{2}=70 \%$ we mostly observe the $\mathrm{P}_{9 \rightarrow 8}$ $(19), P_{10 \rightarrow 9}(17), P_{11 \rightarrow 10}(16), P_{12 \rightarrow 11}(17)$, and $P_{14 \rightarrow 13}(15)$ transitions.

So in this case, first, there are a lot of potentially lasing levels for which the gain is not too far below the threshold value and, second, less vibrational energy present in potentially lasing levels is emitted as stimulated radiation.

It was with this configuration that up to now we found more than 70 vibrational-rotational transitions by tuning the grating at the proper angle and distance from the $70 \%$ reflection window.

In table 1 all these measured transitions are shown ranging from $5.2 \mu \mathrm{m}$ up to about $6.1 \mu \mathrm{m}$ (which is from $1900 \mathrm{~cm}^{-1}$ down to $1640 \mathrm{~cm}^{-1}$ ). The missing transitions are probably a result of atmospheric ab- sorptions. The atmospheric path length from the outcoupling mirror to the detector was about $4 \mathrm{~m}$. The output power of the different lines varied between 25 $\mathrm{mW}$ and $2 \mathrm{~W}$. This can probably be more for some strong lines if the losses in the cavity are made still higher, so that no transition can oscillate without the grating.

Conclusion. Although the wavelength-selective cavity we investigated was not ideal as the outcoupling losses were not high enough, we yet obtained valuable information about the possibilities of our three mirror wavelength-selective system for the $\mathrm{CO}$ laser. We suggest that by decreasing the reflectivity of mirror $\mathrm{M}_{2}$ the laser will oscillate on only one line and that even more transitions should be possible. The stability of the laser is good. If there are fluctuations, they are mostly very slow. However, it might be possible to avoid them by using a feed-back controlled piezoelectric scanner.

\section{References}

[1] P.J.M. Peters, W.J. Witteman and R.J. Zuidema, Appl. Phys. Lett. 37 (1980) 119.

[2] G.J. Ernst and W.J. Witteman, IEEE J. Quant. Electron. QE-7 (1971) 484.

[3] M.L. Bhaumik, W.B. Lacina and M.M. Mann, IEEE J. Quantum. Electron. QE-8 (1972) 150.

[4] P.J.M. Peters, Ph.D. Thesis, Twente University of Technology (1981). 